\title{
A DECOMPOSITION OF A MEASURE SPACE WITH RESPECT TO A MULTIPLICATION OPERATOR
}

\author{
JAMES S. HOWLAND ${ }^{1}$
}

\begin{abstract}
Let $A$ be a bounded multiplication operator on $L_{2}(\Omega, m)$, where $\boldsymbol{\Omega}$ is a complete separable metric space and $m$ a Borel measure. A set of measure zero can be removed from $\Omega$ so that the multiplicity function of $A$ is equal to the cardinality of the preimage. In the proof, $\Omega$ is decomposed into subsets of simple multiplicity.
\end{abstract}

Let $(\Omega, m)$ be a separable measure space and $\alpha(x) \in L_{\infty}(\Omega, m)$. This article concerns the spectral multiplicity theory of the normal operator $A u(x)=$ $\alpha(x) u(x)$ on $\mathcal{H}=L_{2}(\Omega, m)$. Although it is not difficult to see that the measure $\nu(E)=m\left(\alpha^{-1}(E)\right)$ is a suitable scalar spectral measure, the multiplicity function $n(z)$ is less accessible. The first naive guess is that

$$
n(z)=\# \alpha^{-1}(\{z\})
$$

where, for any set $E, \# E$ is the cardinality of $E$ if $E$ is finite, and $\infty$ otherwise. However, this formula fails even for continuous functions on $\boldsymbol{\Omega}=[0,1][1$, p. 854, Example D]. Abrahamse and Kriete [1] have shown that (*) is correct if the preimage $\alpha^{-1}(\{z\})$ is replaced by an "essential" preimage, which they define. One might also ask, however, whether (*) is correct as it stands after a suitable set of $m$-measure zero is removed from $\Omega$. It will be shown below that this is indeed true if $\Omega$ is a complete separable metric space, and $m$ a Borel measure.

First, however, the following question must be answered. According to multiplicity theory, one obtains a decomposition

$$
L_{2}(\Omega, m)=\sum_{k=1}^{\infty} \oplus \mathcal{H}_{k}
$$

where each $\mathcal{H}_{k}$ is a reducing subspace of $A$ on which $A$ has simple multiplicity. To what extent can one achieve this decomposition of $L_{2}(\Omega, m)$ by decomposing $\Omega$ ? More precisely, can one always choose $\mathcal{H}_{k}=L_{2}\left(E_{k}, m\right)$ for suitable subsets $E_{k}$ of $\Omega$ ? It will be shown that the function $\alpha(x)$ induces an essentially unique decomposition of $\Omega$ into disjoint sets $E_{0}$ and $F_{0}$ such that (i) the decomposition of $L_{2}\left(E_{0}, m\right)$ in terms of sets $E_{k}$ is possible, while (ii) the restriction of $A$ to any subset of $F_{0}$ has finite, positive multiplicity $\nu$-almost

Received by the editors May 2, 1978 and, in revised form, March 16, 1979.

AMS (MOS) subject classifications (1970). Primary 47B15; Secondary 47A65.

Key words and phrases. Multiplication operator, spectral multiplicity.

'Partly supported by NSF Grant MCS-76-06427A01.

(C) 1980 American Mathematical Society 0002-9939/80/0000-0068/\$02.00 
nowhere. The theorem stated above follows from this decomposition and an inequality of [1].

The author is pleased to acknowledge conversations with T. L. Kriete.

The complex numbers are denoted by $\mathbf{C}$, and real $n$-space by $\mathbf{R}^{n}$. By $\nu \gg \mu$ is meant that $\mu$ is absolutely continuous with respect to $\nu$.

1. A decomposition theorem. For any measurable subset $E$ of $\Omega$, define $A \mid E$ to be the restriction of $A$ to its reducing subspace $L_{2}(E, m)$. A normal operator with scalar spectral measure $\nu$ will be said to have uniform multiplicity $N$ on a subset $\Gamma$ of $\mathbf{C}$ iff its multiplicity function is identically equal to $N$ on $\Gamma, \nu$-a.e. The multiplication operator $A$ will be said to have uniform multiplicity $N$ on a subset $E$ of $\Omega$ iff $A \mid E$ has uniform multiplicity $N$ on $\mathbf{C}$.

Let $A=\int z d E(z)$. If $\phi \in L_{2}(\Omega, m)$, and $d \mu(z)=d\langle E(z) \phi, \phi\rangle$, then

$$
\int p(z, \bar{z}) d \mu(z)=\left\langle p\left(A, A^{*}\right) \phi, \phi\right\rangle=\int p(\alpha(x), \overline{\alpha(x)})|\phi(x)|^{2} d m(x),
$$

which implies that

$$
\mu(\Gamma)=\int_{\alpha^{-1}(\Gamma)}|\phi(x)|^{2} d m(x)
$$

for any Borel set $\Gamma \subset \mathbf{C}$.

It follows easily that $\nu(S)=m\left(\alpha^{-1}(S)\right)$ is the scalar spectral measure of $A$. For if $\nu(\Gamma)=m\left(\alpha^{-1}(\Gamma)\right)=0$, then $\mu(\Gamma)=0$ for every $\phi$; and, conversely, if $\mu(\Gamma)=0$ for every $\phi$ then $\alpha^{-1}(\Gamma)$ must have $m$-measure zero, which means that $\nu(\Gamma)=0$.

Equation (1.1) also shows that $E[\Gamma] \mathcal{H}=L_{2}\left(\alpha^{-1}(\Gamma), m\right)$ so that $A \mid \alpha^{-1}(\Gamma)$ has scalar spectral measure $\nu_{\Gamma}(S)=\nu(S \cap \Gamma)$. Moreover, if $A$ has uniform multiplicity $N$ on $\Gamma$, then $A \mid \alpha^{-1}(\Gamma)$ has uniform multiplicity $N$. Thus if $A \mid S$ has finite positive multiplicity $N$ on any set $\Gamma \subset \mathbf{C}$, one can find a subset of $S$-namely $S \cap \alpha^{-1}(\Gamma)$-on which $A$ has uniform multiplicity $N$.

LEMMA 1. If $A$ has finite nonzero multiplicity on $S \subset \Omega$ then $S$ has a subset $E$ of positive measure on which $A$ has simple multiplicity.

Proof. One may assume that $S$ has finite measure and, by the preceding remark, that $A$ has uniform multiplicity $N$ on $S$. If $N>1$, there is a nonzero vector $\psi$ orthogonal to the span of $\chi, A \chi, A_{\chi}^{2}, \ldots$, where $\chi(x)=\chi_{s}(x)$. Equivalently,

$$
\int_{S} \psi(x) F(\alpha(x)) d m(x)=0
$$

for every bounded Borel function $F$. Taking $F(\lambda) \equiv 1$ shows that both $S_{+}=\{x \in S \mid \psi(x)>0\}$ and $S_{-}=\{x \in S \mid \psi(x) \leqslant 0\}$ must have positive $m$-measure. Let $A_{ \pm}=A \mid S_{ \pm}$. There are, a priori, two cases.

(i) Either $A_{+}$or $A_{-}$has multiplicity less than $N$ on some set.

(ii) Both $A_{+}$and $A_{-}$have uniform multiplicity $N$. 
Now in case (ii) the scalar spectral measures $\Gamma_{ \pm}$of $A_{ \pm}$must lie on disjoint sets $\Gamma_{ \pm}$, for on any set $\Gamma$ of positive $\nu_{+}$and $\nu_{-}$-measure, the multiplicity of $A=A_{+} \oplus A_{-}$would be $2 N$. Hence, $L_{2}\left(S_{ \pm}, m\right)=E\left[\Gamma_{ \pm}\right] \mathcal{H}=$ $L_{2}\left(\alpha^{-1}\left(\Gamma_{ \pm}\right), m\right)$ which implies that $\chi_{E_{+}}(x)=\chi_{\alpha^{-1}\left(\Gamma_{+}\right)}(x)=\chi_{\Gamma_{+}}(\alpha(x)) m$-a.e. But then

$$
\int \chi_{\Gamma_{+}}(\alpha(x)) \psi(x) d m(x)=\int_{E_{+}} \psi(x) d m(x)>0
$$

in contradiction to (1.2). Therefore case (ii) cannot occur.

Hence, one can find a subset of $S$, of positive measure, on which the multiplicity of $A$ is less than $N$. Continuation of this process eventually terminates with a set of simple multiplicity.

A subset $F$ of $\Omega$ will be called purely infinite (with respect to $A$ ) iff $A \mid F_{1}$ has uniform infinite multiplicity for every $F_{1} \subset F$ with $m\left(F_{1}\right)>0$.

THEOREM 1. There is an essentially unique maximal purely infinite subset $F_{0}$ of $\Omega$. The complement $E_{0}$ of $F_{0}$ is the disjoint union of sets $E_{1}, E_{2}, \ldots$ on which $A$ has simple multiplicity.

Proof. By Zorn's lemma, there is a maximal family $\mathcal{E}$ of disjoint subsets of $\Omega$, of positive measure, on which $A$ has simple multiplicity. Because $m$ is separable $\mathcal{E}=\left\{E_{1}, E_{2}, \ldots\right\}$ must be countable. Let $E_{0}=E_{1} \cup E_{2}$ $\cup \cdots$ and $F_{0}=\Omega \sim E_{0}$. If $F \subset F_{0}$ and $m(F)>0$, then $A \mid F$ must have uniform infinite multiplicity, for otherwise by the lemma, there would be a subset $F_{1}$ of $F$ on which $A$ would have simple multiplicity, in contradiction to maximality of $\mathcal{E}$. Thus $F_{0}$ is purely infinite.

Moreover, every purely infinite set $F$ is essentially contained in $F_{0}$. For $A \mid\left(F \cap E_{k}\right)$ can have multiplicity no greater than one, so that one must have $m\left(F \cap E_{k}\right)=0$ for every $k$, and hence $m\left(F \cap E_{0}\right)=0$.

Of course, either $E_{0}$ or $F_{0}$ may be equal to $\Omega$. For example, if $m$ has no atoms and $\alpha(x)$ is constant on a set $F$ of positive measure, then $F \subset F_{0}$. Another example will be given at the end of the next section.

It is interesting to inquire how far one can go in duplicating the constructions of measure theory in terms of sets. On $F_{0}$, the answer is clearly "nowhere". However, one can show that $E_{0}$ is the disjoint union of a countable totally-ordered family $\left\{E_{i}\right\}$ of sets such that (a) if $i>j$, then $\nu_{E_{i}} \gg \nu_{E_{j}}$, and (b) $E_{i}$ is a maximal set of simple multiplicity in $E_{0} \sim \cup_{j<i} E_{j}$.

2. The multiplicity function. Let $n(z)$ be the multiplicity function of $A$.

THEOREM 3. If $\Omega$ is a complete separable metric space, there is a subset $\Omega_{0}$ of $\Omega, m\left(\Omega_{0}\right)=0$, such that if $\alpha_{0}(x)$ is the restriction of $\alpha(x)$ to $\Omega \sim \Omega_{0}$, then

$$
n(z)=\# \alpha_{0}^{-1}(\{z\})
$$

$\nu$-a.e. 
Proof. Let $\Gamma$ be the set where $n(z)$ is finite. In general, one has [1, p. 852],

$$
n(z) \leqslant \# \alpha^{-1}(\{z\})
$$

so that (2.1) holds on $\alpha^{-1}(\mathrm{C} \sim \Gamma)$ with $\alpha(x)$ unchanged. Moreover, $\alpha^{-1}(\Gamma)$ is contained in $E_{0}$ and is therefore a countable union of sets of simple multiplicity. The theorem therefore follows from the following lemma.

LEMMA 3. If $A$ has simple multiplicity on $E$, there is a set $F, m(F)=0$, on which $\alpha(x)$ is one-one.

Proof. One may assume that $E$ is closed (hence complete) by replacing $E$ by $\bar{E}$ and $m$ by $\bar{m}(S)=m(S \cap E)$; the $\bar{m}$-nullset $\bar{E} \sim E$ can be dropped at the end. One may also assume that $m(E)<\infty$. Then $1=\chi_{E}(x)$ is cyclic, and the correspondence $\Phi: p(\alpha(x)) \rightarrow p(z)$ for polynomials $p$ defines a unitary mapping $\Phi$ of $L_{2}(\Omega, m)$ onto $L_{2}(C, \nu)$. The restriction of $\Phi$ to characteristic functions is an isomorphism of the measure algebras, and hence $[2, \mathrm{p} .329$, Theorem 12], if a nullset is omitted from $E$, there is a one-one mapping $\phi$ of $E$ onto a Borel subset of $\mathrm{C}$. The function $\phi$ is bounded because $\nu$ has bounded support.

Let $p_{n}(x)$ be a sequence of polynomials such that $p_{n}(\alpha(x)) \rightarrow \phi(x)$ a.e., say on $E \sim F$ where $m(F)=0$. Then $\alpha(x)$ is one-one on $E \sim F$; for if $\alpha\left(x_{1}\right)=$ $\alpha\left(x_{2}\right)$, then $\phi\left(x_{1}\right)=\phi\left(x_{2}\right)$ and hence $x_{1}=x_{2}$.

COROLlaRY 1. If $m(E)=0$ for every Borel set on which $\alpha(x)$ is one-one, then $\Omega=F_{0}$ and $n(z) \equiv \infty$.

For example, let $\Omega$ be an open set in $\mathbf{R}^{n}, n \geqslant 2$,with Lebesgue measure, and $\alpha(x)$ a $C^{1}$ function the gradient of which does not vanish on $\Omega$. By the Implicit Function Theorem, coordinates may be chosen near any point so that $\alpha(x)=x_{1}$, from which it is clear that $\alpha(x)$ is one-one only on sets of measure zero. Hence, in this case $\Omega=F_{0}$ and $n(z) \equiv \infty$.

The referee has pointed out that the following proposition on von Neumann algebras may replace Theorem 1 in the proof of Theorem 2: "If $R$ is an abelian von Neumann algebra having no summand of infinite multiplicity, and $M$ is a maximal abelian subalgebra in the finite type $I$ von Neumann algebra $R^{\prime}$, then $M$ contains an orthogonal sequence $E_{j}$ of abelian projections for $R^{\prime}$ such that $\Sigma E_{j}=1$ ".

\section{REFERENCES}

1. M. B. Abrahamse and T. L. Kriete, The spectral multiplicity of a multiplication operator, Indiana J. Math. 22 (1973), 845-857.

2. H. L. Royden, Real analysis, 2nd ed., Macmillan, New York, 1968.

Department of Mathematics, University of Virginia, Charlottesville, Virginia 22903 